\title{
Intestinal Bacterial Flora that Compete on the Haem Precursor Iron Fumarate in Iron Deficiency Anemia Cases
}

\author{
Samy Abdel Hamid Selim ${ }^{1,2 *}$, Sahar Moustafa El Alfy ${ }^{1}$, Atef Monier Diab ${ }^{1}$, Mohamed Helmy Abdel Aziz ${ }^{1}$ and Mona \\ Farouk Warrad ${ }^{2}$ \\ ${ }^{1}$ Microbiology Laboratory, Department of Medical Laboratory Science, Collage of Applied Medical Science, Al-Jouf \\ University, P.O 2014, Saudi Arabia \\ ${ }^{2}$ Microbiology Section, Botany Department, Faculty of Science, Suez Canal University, P.O 41522, Ismailia, Egypt \\ E-mail: sadomm2003@yahoo.com
}

Received 24 December 2011; received in revised form 30 January 2012; accepted 27 February 2012

\begin{abstract}
Aims: The study focused on finding if there is any possible relation between the intestinal bacterial population quantitative and qualitative and the deficiency of the most important iron compounds as haem precursors.

Methodology and Results: Blood complete picture and stool analyses were done to 750 volunteer cases whom were asked for these analyses by their physicians. Analyses proved that 560 cases representing $75.2 \%$ were anemic as the $\mathrm{RBC}(\mathrm{s})$ based on counts of the total studied cases of less than $263 \times 10^{4}$ and the haemoglobin amount ranged between 7.2 and $11.3 \mathrm{~g} / \mathrm{dl}$, while the remainder $24.8 \%$ of the volunteer sample was not anemic. A high male/female ratio of anemic cases, 1:27 was also documented. Considering that all the studied stool samples should be completely free from any parasites or any other anemia-related diseases was a priority. Bacteriological analysis of stool samples of the anemic cases resulted in the detection of high counts of total viable bacteria, exceeded $42 \times 10^{9} \mathrm{cfu} / \mathrm{g}$, while it was never more than $26 \times 10^{6} \mathrm{cfu} / \mathrm{g}$ and decreased to $4 \times 10^{6} \mathrm{cfu} / \mathrm{g}$ in many cases in this study. Identifying of the 361 bacterial isolates, were found to belong to 12 genera and 19 species, 6 of them; Pseudomonas putrefaciens, Micrococcus luteus, Erysipelothrix rhusiopathiae, Bacillus megaterium, Bacillus pumilus and Bacillus coagulans, were found and in high counts in the stool samples of only anemic cases. The ability of these isolates to compete for iron compounds such as ferrous fumarate alone or with glucose and phytate as activators or inhibitors to these abilities was investigated. Results proved 11 species out of the 19 identified species are capable to use and compete on ferrous fumarate as a haem precursor. Sensitivity test for the representatives of the 19 species and 6 of the most commonly used antibiotics in the Egyptian pharmacy, using standard disc method, revealed variable susceptibilities of almost all of them to more than one of the studied antibiotics, except Corynebacterium equatium, which was found very resistant to two antibiotics; colistin sulfate and erythrocin.
\end{abstract}

Conclusion, significance and impact of study: The study finally concluded the strong role of intestinal bacterial counts and types as competitors on the haem precursor iron-containing compounds like ferrous fumarate.

Keywords: Intestinal Bacterial Flora, Haem Precursor, Iron Fumarate, Iron Deficiency Anemia.

\section{INTRODUCTION}

Anemia is one of the more common blood disorders. Anemia can be defined as having less than the normal number of red blood cells or less hemoglobin than normal in the blood. There are several types of anemia and the most common type of anemia is iron deficiency anemia that happens when there is a deficiency of iron in the body. Iron deficiency anemia occurs when a person loses blood from problems such as heavy periods, ulcers, colon polyps, or colon cancer. In addition, a diet that does not have enough iron in it can also cause iron deficiency anemia (Hallberg, 1982; Layrisse et al., 2000). Iron is essential to all microorganisms. To obtain iron from the very low concentrations present in their environment, microorganisms have developed sophisticated mechanisms such as the siderophore system (Bagg and Neilands, 1987; Crichton and Ward, 1992). Anemia is seen in the setting of infectious, inflammatory, and neoplastic diseases. It results, from changes in the intracellular metabolism of iron. Alterations of iron physiology seen in many clinical circumstances make excess iron available to microorganisms, thus enhancing their pathogenicity. Nearly all groups of potentially pathogenic bacteria thus far examined require iron for activation of ribonucleotide reductase, aconitase and various enzymes involved in electron transfer and oxygen metabolism (Bullen et al., 1978; Byers, 1987; Griffiths, 1987; 1991; Martinez et al., 1990; Braun et al., 1998).

The intestinal bacterial flora in patients with anemia has long attracted the interest of investigators, but few recent studies have been reported. The present investigation was undertaken to reappraise the significance of the bacterial flora of the small intestine and to correlate bacterial growth with intestinal absorption in patients with 
is iron deficiency anemia. The implication of intestinal bacteria in the pathogenesis of anemia began with the intestinal-toxin theories of William Hunter in 1900. There followed a long series of investigations of the gastrointestinal flora in anemia with successful attempts to demonstrate excesses of specific types of bacteria in this disease. Davidson (1928) studied the bacterial flora of the stomach and colon and found marked quantitative increases of microorganisms in these areas in patients with anemia. Although the upper gastrointestinal flora was frequently colonic in type, no particular organism was consistently present. Other investigators have noted a proliferation of fecal organisms in the upper gastrointestinal tract of patients with anemia (Conrad and Crosby, 1963; Willam et al., 1964).

The present study focused on determining the quantitative and qualitative variation of the intestinal bacterial population in relation with the iron deficiency anemia features in Egypt. Also the study investigated the possibility of prescribing antibacterial agents along with other anemia-treating drugs.

\section{MATERIALS AND METHODS}

\section{Patients}

A well-taken history from somebody close to the patient, as well as from the actual patient, particularly when there is doubt as to whether he is capable, or willing to give full details of diet, drug ingestion, was carefully and accurately recorded for every single case. Random survey of 750 volunteer cases in order to determine anemic cases and choose the appropriate study sample was run in hospitals and health centres of Ismailia city, Egypt. Iron-deficiency anemia was defined as a hemoglobin concentration less than $14 \mathrm{~g} / \mathrm{L}$ for men and less than $12 \mathrm{~g} / \mathrm{L}$ for women (Marignani et al., 1999), a mean corpuscular volume less than $80 \mathrm{fL}$, and a serum ferritin level less than $30 \mathrm{mg} / \mathrm{L}$. Outpatients with an obvious cause of blood loss, such as a heavy menstrual period, epistaxis, active gastrointestinal hemorrhage or evidence of fecal occult blood positivity were excluded from the study. Other exclusion criteria were gastrointestinal or hematologic cancer at the time of observation, chronic renal failure, severe cardiopulmonary disease, reported or suspected pica, hemolysis, aplastic anemia, alcoholism or liver cirrhosis, and pregnancy. A hospital dietitian excluded an iron-poor diet as a cause of iron deficiency anemia.

\section{Measurements}

Whole blood specimens for haematology laboratory were collected by venepuncture technique (NCCLS 1992a). The syringe and needle method were followed as it is preferred when difficulty in drawing the specimen is anticipated, e.g. patients with fragile or hardened vein walls. Gentle inversion, five to ten times of the tube containing part of the blood sample and the anticoagulant, trisodium citrate $\left(\mathrm{Na}_{3} \mathrm{C}_{6} \mathrm{H}_{5} \mathrm{O}_{7}\right)$ recommended in a 1:4 anticoagulant/blood ratio for determining the erythrocyte sedimentation rate (ESR) (ICSH, 1977). EDTA $\left(\mathrm{C}_{10}\right.$ $\mathrm{H}_{16} \mathrm{~N}_{2} \mathrm{O}_{8}$ ) was added to the rest of the sample at 3.7-5.4 $\mu \mathrm{mol} / \mathrm{mL}$ for performing other blood analyses including; haemoglobin value, red blood cell count, white blood cell count, mean corpascular volume, mean corpascular haemoglobin and mean corpascular haemoglobin concentration (Lewis and Koepke, 1995).

\section{Microbiological Analysis}

Two stool samples volume of at least 1 teaspoonful $(5 \mathrm{~mL})$ was collected in clean, waxed cardboard or plastic container and another in sterile glass vial for bacteriological studies and delivered to the laboratory with no longer than one hour for physical and microbiological examinations. The first was microscopically examined for parasites. One gram of the second was suspended in 100 $\mathrm{mL}$ sterile saline, serially diluted and $1 \mathrm{~mL}$ of the dilutions was cultivated on nutrient agar plates using the pour plate method. TVB counts cfu/mL were the recorded after $24 \mathrm{~h}$ incubation at $37{ }^{\circ} \mathrm{C}$. Macro and micro-morphological descriptions as well as complete identification of the bacterial isolates following the biochemical charts (Holt, 1994) were achieved. Sensitivity test for all isolates was performed against six antibiotics namely; colistin sulpfate, streptomycin, velosef, chloramphenicol, ampicillin and erythromycin using standard antibiotic discs (Murray et al., 1995).

\section{Utilization of Haem Precursor Compounds}

For the detection and estimation of bacterial isolates that compete for fumarate as a haem precursor, pure bacterial cultures were grown on nutrient agar for $24 \mathrm{~h}$ at $37^{\circ} \mathrm{C}$, then harvested and suspended in Davis minimal broth medium. Growth was measured and equally adjusted for all cultures absorbance spectrophotometrically at $A_{600}$. $A$ set of four tubes was prepared as follows for each bacterial isolate; the first contained $1 \mathrm{~mL}$ bacterial suspension and $4 \mathrm{~mL}$ of the minimal broth, the second was the same as the first supplemented with ferrous fumarate $1 \mu \mathrm{g} / \mathrm{mL}$, the third was the same as the second plus phytic acid $7.3 \mu \mathrm{g} / \mathrm{mL}$ and the fourth was the same as the second plus glucose $7.3 \mu \mathrm{g} / \mathrm{mL}$. In a shaker incubator, all tube sets were incubated at $37^{\circ} \mathrm{C}$ for $48 \mathrm{~h}$ and the absorbance was read again at $A_{600}$. A standard curve was plotted and the extent of growth was directly determined from its absorbance.

\section{RESULTS}

Volunteer sample size covered in this study started with 750 cases. Non anemic cases (186) comprised $24.8 \%$ of the total sample size, $9 \%$ of these non-anemic cases were infected with parasites. Anemic cases (560) comprised $75.2 \%$ of the total sample size were suffering from obvious anemia symptoms, parasite- free and had no clearly-defined suspected cause of anemia. Healthy nonanemic control 107 volunteers were as fully as the anemic 
cases. Results of complete blood picture and parasitological stool analyses were the tools upon which samples were described and the study was designed. The sample included 448 females and 112 males with haemeoglobin value $(\mathrm{Hb})$, ranged from 7.2-11.7 for females and 11.3 for males, while control $(\mathrm{Hb})$ values were above 12 up to 17.4 represented by 36 females and 71 males volunteers. Counts of RBC(s) ranged from $263 x$ $10^{4}$ to $707 \times 10^{4}$. The erythrocyte sedimentation rate (ESR) ranged from $4-110$ after $1 \mathrm{~h}$ and from 11 to 110 after $2 \mathrm{~h}$. White blood cell counts ranged between 2625 and 11650. Other blood picture components such as P.C.V, M.C.V, M.C.H and M.C.H.C were examined and the results were not published.

Enumeration of bacteria in stool samples of the studied anemic cases showed high total viable bacterial counts (TVB) counts and great variations from sample to sample. A minimum of $13 \times 10^{6} \mathrm{cfu} / \mathrm{g}$ and a maximum of $42 \times 10^{9}$ $\mathrm{cfu} / \mathrm{g}$ were recorded for anemic cases, both from females, while in the male sample TVB count was $75 \times 10^{6} \mathrm{cfu} / \mathrm{g}$. The counts for control volunteer samples started from $4 \mathrm{x}$ $10^{6}$ (a female case) up to $26 \times 10^{6}$ (a male case). Bacterial isolates from stool samples (361 isolates) were identified and confirmed to belong to 12 genera and 19 species. Neisseria mucosa (190 isolates) comprised the highest percentage $(52.6 \%)$ of the total isolates as well as the highest distribution rate amongst all cases including control volunteers. In the second place came $P$. pseudoalcaligenes and $P$. putrefaciens (45 isolates) and they detected only in anemic cases. In addition to $P$. pseudoalcaligenes. More six species belong to four genera were detected only in stool samples of anemic cases namely, B. pumilus, B. megaterium, B. coagulans, E. rhusiopathiae, M. luteus, and $A$. viridans. Species those detected both in anemic and non-anemic stool specimens were dominated by $N$. mucosa with other eleven species namely; L. monocytogenes, $S$. aureus, $B$. cereus, $B$. subtilis, $P$. mirabilis, $A$. punctata, $A$. calcoaceticus, $M$. rosus, $C$. equatium and $C$. pseudotuberculosis.

Representatives of the identified species (19 isolates) were chosen to perform sensitivity test against six commonly used antibiotics. Erythrocin suppressed the growth of $N$. mucosa, $L$ monocytogenes, $M$. luteus, $P$. pseudoalcaligenes, $B$. pumilus and $E$. rhusiopathiae. Streptomycin inhibited the growth of $B$. coagulans, $B$. megaterium, $B$. cereus, $A$. viridans, $C$. pseudotuberculosis, $P$. putrefaciens and $M$. luteus. Chloramphemical suppressed the growth $S$. aureus, $A$. punctate, $M$. roseus, $M$. luteus, $P$. mirabilis, $A$. calcodceticus, $B$. megaterium and $A$. viridans. These three antibiotics seemed to be broad spectrum and have a quite wide range against most of the studied bacterial isolates and showed clear zones from 17 to $25 \mathrm{~mm}$ in diameter. The $N$. mucosa isolate was sensitive to all the six antibiotics and was highly susceptible to chloramphenicol, ampicillin and erythrocin having clear zone diameters of 10,11 and $12 \mathrm{~mm}$ respectively. L. monocytogenes was more or less equally affected by all the studied six antibiotics. M. luteus showed higher susceptibility to all the studied antibiotics than $M$. roseus. $P$. pseudoalcaligenes showed high sensitivity to velosef and eryrthrocin, while $P$. putrefaciens strongly affected by streptomycin and ampicillin. Genus Bacillus showed variable susceptibility profiles. The tested five species were sensitive to all the studied antibiotics with high tendency of $B$. subtilis to streptomycin and velosef, $B$. pumilus to erythrocin, $B$. megaterium and $B$. cereus to chloramphenicol and $B$. coagulans to streptomycin. C. equatium was greatly affected by velosef and chloramphenicol, while showed complete resistance to colistin sulfate and erythrocin.

All the identified nineteen bacterial species isolated from stool samples of anemic cases and control volunteers were examined for their capability in using iron in the form of ferrous feumarate (FeFu) alone and in combination with phytate (ph) and/ or glucose (G). Three types of controls were necessary one without supplements, a second with only glucose $(G)$ and a third with only phytate (ph). All the investigated isolates representing the 19 identified species took up iron in the form of (FeFu) but 10 of them namely; $N$. mucosa, A. punctata, M. roseus, M. luteus, $P$. mirabilis, $P$. putrefaciens, C. equatium, B. pumilus, B. megaterium $B$. coagulans, and $E$. rhusiopathiae consumed much more iron in the form of (FeFu) than the representative isolates of the other 9 species and gave relatively high absorbance readings ranged between $0.14-0.2$ (Table 1). They showed enhanced usage of (FeFu) when combined with $(\mathrm{ph})$ and with $(\mathrm{G})$. The other 9 isolates showed variable responses in which, for example, (ph) and (G) acted as growth limiting factors as the bacterial growth solely on them was greater than in combinations with (FeFu).

Genus of Neisseria was presented by only one species; mucosa. It showed very little growth in the presence of $(\mathrm{FeFu})$ alone and $(\mathrm{G})$ alone, while the growth was very heavy in a combination of them both. Growth in (ph) alone was as the same as in a combination of (FeFu+ph). Genus Staphylococcus was also presented by only one species; aureus. The resulted growth rate was the same in $(G)$ alone and $(F e F u+G)$. Same results were recorded for (ph) alone and for (FeFu+ph). The four growth rate readings were high if compared with the very low reading of growth rate with (FeFu) alone. Species monocytogenes the only representative for genus Listeria, showed scanty growth in $(\mathrm{FeFu})$ alone and in $(\mathrm{FeFu}+\mathrm{G})$ while it gave rich growth with $(\mathrm{G})$ alone. The growth in (ph) alone and with (FeFu+ph) did not show any difference. A big difference was recorded between the growth rate of $A$. punctata in the presence of a combination of $(\mathrm{FeFu}+\mathrm{G})$, in one hand and the growth in (FeFu) alone or in (G) alone on the other hand, the former was much higher. No obvious difference was detected between growths in (ph) alone and in (FeFu+ph). A little bit better growth was observed in $(\mathrm{FeFu}+\mathrm{G})$ than in $(\mathrm{G})$ alone or $(\mathrm{FeFu})$ alone for the only species; mirabilis that presented genus Proteus. No growth differences were recorded in (FeFu+ph) than in (ph) alone. 
Table 1: Growth after $48 \mathrm{~h}$ of bacterial isolates on non supplemented minimal broth medium as control and on minimal broth medium supplemented with ferrous fumarate (FeFu), glucose $(G)$, ferrous fumarate + glucose $(F e F u+G)$, phytate (ph) and with ferrous fumarate + phytate (FeFu+ph).

\begin{tabular}{lcccccc}
\hline \multicolumn{1}{c}{ Bacterial isolates } & Control & FeFu & G & FeFu+G & ph & FeFu+ph \\
\hline A. calcoaceticus & 0.012 & 0.076 & 0.087 & 0.118 & 0.152 & 0.019 \\
A. viridans & 0.01 & 0.007 & 0.049 & 0.114 & 0.129 & 0.13 \\
A. punctata & 0.01 & 0.002 & 0.012 & 0.119 & 0.112 & 0.126 \\
B. subtilis & 0.035 & 0.071 & 0.099 & 0.076 & 0.155 & 0.066 \\
B. coagulans & 0.01 & 0.006 & 0.011 & 0.15 & 0.185 & 0.125 \\
B. megaterium & 0.023 & 0.008 & 0.073 & 0.091 & 0.077 & 0.092 \\
B. pumilus & 0.022 & 0.007 & 0.098 & 0.181 & 0.084 & 0.021 \\
B. cereus & 0.016 & 0.015 & 0.011 & 0.026 & 0.024 & 0.011 \\
C. equatium & 0.098 & 0.01 & 0.145 & 0.153 & 0.111 & 0.018 \\
C. pseudotuberculosis & 0.01 & 0.01 & 0.104 & 0.122 & 0.113 & 0.136 \\
E. rhusiopathiae & 0.018 & 0.02 & 0.115 & 0.131 & 0.12 & 0.134 \\
L. monocytogenes & 0.023 & 0.011 & 0.164 & 0.011 & 0.103 & 0.106 \\
M. leteus & 0.017 & 0.017 & 0.084 & 0.145 & 0.11 & 0.125 \\
M. roseus & 0.016 & 0.016 & 0.157 & 0.136 & 0.149 & 0.163 \\
N. mucosa & 0.017 & 0.015 & 0.025 & 0.141 & 0.127 & 0.0134 \\
P. putrefaciens & 0.01 & 0.008 & 0.105 & 0.13 & 0.116 & 0.152 \\
P. pseudoalcagenes & 0.019 & 0.011 & 0.097 & 0.113 & 0.098 & 0.015 \\
P. mirabilis & 0.017 & 0.018 & 0.102 & 0.135 & 0.119 & 0.125 \\
S. aureus & 0.018 & 0.014 & 0.118 & 0.12 & 0.1 & 0.115 \\
\hline
\end{tabular}

Genus Pseudomonas had two representative species; pseudoalcaligenes and putrefaciens. Scanty growth was noticed for $P$. pseudoalcaligenes in (FeFu+ph) while growth was high in $(\mathrm{G})$ alone, $(\mathrm{FeFu}+\mathrm{G})$ and $(\mathrm{ph})$ alone. For $P$. putrefaciens growth rate in combinations of $(\mathrm{FeFu}+\mathrm{G})$ and $(\mathrm{FeFu}+\mathrm{ph})$ was higher than in $(\mathrm{G})$ or $(\mathrm{ph})$ alone. A. calcoaceticus gave slightly higher growth in $(\mathrm{FeFu}+\mathrm{G})$ than in $(\mathrm{G})$ alone, but gave very poor growth in (FeFu+ph) if compared with the growth in (ph) alone. Genus Corynebacterium had two representative species in the present work; equatium and pseudotuberculosis. Growth of both in (FeFu) was much lower than in the control. The rate of growth increased, almost the same amount of increase, in (G), (ph) and in (FeFu+G) than in the control. $A$. viridans was the only species that gave negative growth reading (growth inhibited) with (FeFu) alone, high reading with $(G)$ alone and higher readings with $(\mathrm{FeFu}+\mathrm{G}),(\mathrm{FeFu}+\mathrm{ph})$ and $(\mathrm{ph})$. E. rhusiopatiae had nearly no changes in growth figures with different treatments.

\section{DISCUSSION}

None of the identified bacterial species in healthy control samples could be recognized as characterizing species for only non-anemic people, as they all were recorded in the studied anemic cases. The presence of certain bacterial species both in anemic and non-anemic control samples was observed, but the counts of each was higher in anemic than in non- anemic cases. The presence of other species of bacteria only in anemic and not in nonanemic samples may pinpoint a possible active competing role of these bacteria or preventing the absorption of ironcontaining haem precursors. $P$. pseudoalcaligenes and $P$. putrefaciens (45 isolates), for example were detected only in anemic cases and not in volunteer control samples. The reciprocal relation obtained between the HB and ESR values, as well as the linear relation that was observed between ESR values and TVB counts in stool samples is a remarkable finding. Bacterial toxins are a well-known cause of high ESR values. This triagonal relation thought to be an indirect relation by Cotran et al. (1999) but when there is no clear clinical reason for anemia, this relation should be considered direct and important. In this study, for example, the lowest investigated figure of hemoglobin was HB 7.2, had ESR values of 110 after both 1 and 2 hours and found to contain TVB counts as high as $8298 \mathrm{x}$ $10^{6} \mathrm{cfu} / \mathrm{g}$ of stool sample. The anemic case with $\mathrm{HB}$ value of 9.5 and ESR reading of 25, 50 after 1, 2 hours respectively, found to harbor TVB counts of only $40 \times 106$ cfu/g of stool sample. The control case with HB value of 13 and ESR readings of 2, 4 after 1,2 hours respectively, proved to have only $9 \times 10^{6} \mathrm{cfu} / \mathrm{g}$ of stool sample. These results confirmed the discussed correlation.

The recorded, more or less far, two points from this relation in this study could be not due to the quantity of the bacteria but due to the quality of them. In the anemic case, for example, that had $\mathrm{BH}$ value of 7.8 and relatively low ESR reading of 18, 40 after 1, 2 hours respectively, showed almost the highest TVB count $31767 \times 10^{6} \mathrm{cfu} / \mathrm{g}$ of stool sample. According to the previously explained relation, it was supposed to have ESR values more than 110. This sample had a wider range of the bacterial population than other studied samples; more than those detected in the stool sample of the case with $\mathrm{HB}$ value of 7.2, but lacking the presence of one important species, $A$. punctata. Many species of this genus have been long documented as human intestine-inhabitants (Agger et al., 1985; Moyer 1987, Challapalli et al., 1988; De la Morena et al., 1993). Out of the identified bacteria in this study 6 
species were detected only in stool samples of anemic cases; i.e. not detected in control stool samples. These 6 species namely; $P$. putrefaciens, $M$. luteus, $E$. rhusiopathiae, $B$. megaterium, $B$. pumilus and $B$. coagulans, are also noticed to found in low HB and high TVB counts. E. rhusiopathiae for example isolated among TVB counts of $1516 \times 10^{6} \mathrm{cfu} / \mathrm{g}$ from anemic case with HB of $9.5 \mathrm{~g} / \mathrm{dl}$. These six species were found sensitive to almost all the tested antibiotics; this indicates how easy their control is. Then the prevention of their suggested counterpart in competing for the iron compounds in normal human intake will diminish to a minimum and, in turn, the need for supplemental iron medication could be avoided. $C$. equatium was the only isolate that showed resistance to two; colistin sulfate and erythrocin, of the six tested antibiotics in this study, while, $C$. pseudotuberculosis was not.

\section{CONCLUSION}

This research addressed a new scope of an old problem. The study confirmed the hypothesized relation between the intestinal population of bacteria both, quantitative and qualitative, and the symptoms of iron deficiency anemia through the possible active competition mechanisms of bacteria on iron compounds as heme precursors. The study recommends the necessity of a culture and sensitivity test to be done for the iron-deficiency anemic patients in Egypt. Prescription of the appropriate antibiotic well then is effective in minimizing the need for supplemental iron medications.

\section{REFERENCES}

Agger, W. A., Maccormick, J. D. and Gurwith, M. J. (1985). Clinical and microbiological features of Aeromonas hydrophila associated diarrhea. Journal Clinical Microbiology, 21, 909-913.

Bagg, A. and Neilands, J. B. (1987). Molecular mechanism of regulation of siderophore mediated iron assimilation. Microbiol 51, 509-518.

Braun, V., Hante, K. and Koster, W. (1998). Bacterial iron transport: mechanisms, genetics, and regulation. Met lons Biol Syst, 35: 67-145.

BULLEN J. J., ROGERS H. J., GRIFFITHS E.: Role of iron in bacterial infections. Curr. Top. Microbiol. Immunol., 80,1-35 (1978).

Byers B.R. (1987). Pathogenic iron acquisition . Life Chem Rept 4:143-159.

Challapalli, M., Tess, B. R., Cunningham, D. G., Chopra, A. K. and Houston, C. W.(1988). Aeromonas associated diarrhea in children. Pediatr Infect Dis, 17: 693-698.

Conrad, M. E. and Crosby, W. H. (1963). Intestinal mucosal mechanisms controlling iron absorption. Blood 22: 406-415.

Cotran, R. S., Kumar, V. and Collins, T. (1999). Pathological Basis of Disease. Sixth Edition, Academic Press.
Crichton, R. and Ward R.G.(1992). Iron metabolism-new perspectives in view. Biochemistry, 31: 11255-11264.

Davidson, L. S. P. (1928). The gastrointestinal flora in pernicious anaemia. J Path and Bact 31: 557-559.

DE LA MORENA M. L., VAN R., SINGH K., BRIAN M., MURRY B. E., PICKERING L. K.: Diarrhea associated with Aeromonas species in children in day care centers. J. Infect. Dis., 168, 215-218 (1993).

Griffiths, E. (1987). The iron-uptake systems of pathogenic bacteria, p. 69-137. In J. J. Bullen and E. Griffiths (ed.), Iron and infection. John Wiley \& Sons, Inc., New York, N.Y.

Griffiths, E. (1991). Iron and bacterial virulence-a brief overview. Boil Met, 4: 7-13.

Hallberg, L. (1982). Iron absorption and iron deficiency. Hum. Nutr Clin Nutr, 126, 373-379.

Holt, J. G., Krieh, N. R., Sneath, P. H., Staley, J. and Williams, S. T.(1994). Bergey's manual of determinative bacteriology. $9^{\text {th }}$ ed. Williams and Wilkins, Co., Baltimore, Md.

Hunter, R. W. (1900). Pernicious Anaemia. Charles Griffin. London.

ICSH (1977). Recommendation for measurement of erythrocyte sedimentation rate of human blood. Am J Clin Pathol, 66: 505-507.

Layrisse, M., Garcia-Csal, M. N., Solano, L., Baron, M. A., Arguell, O. F., Lioverad, D., Ramirez, J., Leets, I. and Tropper, E. (2000). Iron bioavailability in humans from breakfasts enriched with iron BisGlycine chelate, Phytates and Polyphenols. American Society for Nutritional Sciences, 130: 2195-2199.

Lewiis, S. M. and Koepke, J. A. (1995). Hematology Laboratory Management and Practice. $1^{\text {st }}$ edition, 37. 65.

Marignani, M., Delle Fave, G., Mecarocci, S., Bordi, C., Angeletti, S., D'ambra, G. 2 (1999). High prevalence of atrophic body gastritis in patients with unexplained microcytic and macrocytic anemia: a prospective screening study. Am. J. Gastroenterol., 94: 766-7.

Martinez, J. L., Delgado-Iribrren, A. and Baguero, F. (1990). Mechanism of iron acquisition and bacterial virulence. FEMS Microbiol Rev 75: 45-56.

Moyer, N. P. (1987). Clinical significance of Aeromonas species isolated from Patients with diarrhea. J Clin Microbiol, 25: 2044-2048.

Murray, P. R., Baron, E. J., Phaller, M. A., Tenover, F. C. and Yolken, R. H. (1995). Manual of Clinical Microbiology. $6^{\text {th }}$ edition, ASM press, Washington, D. C.

National Committee For Clinical Laboratory Standards (NCCLS) (1992A). Percutaneous collection of arterial blood for laboratory analysis. Approved Standard Villanova, PA.

Willam, C. S., Frannz, M. D., Farid, I. H. and Wilier, W. M. D. (1964). Studies of the small-intestinal bacterial flora and of intestinal absorption in Pernicious Anemia. American J of Digestive Diseases, 9: 416425. 